\title{
Evaluated displacement and gas production cross-sections for materials irradiated with intermediate energy nucleons
}

\author{
Alexander Yu. Konobeyev ${ }^{1, \text { a }}$, Ulrich Fischer ${ }^{1}$, Pavel E. Pereslavtsev ${ }^{1}$, Stanislav P. Simakov ${ }^{1}$, and Sibel Akça ${ }^{2}$ \\ ${ }^{1}$ Institute for Neutron Physics and Reactor Technology, Karlsruhe Institute of Technology, 76344 Eggenstein-Leopoldshafen, Germany \\ 2 Çukurova Univercity, 01330 Adana, Turkey
}

\begin{abstract}
Atomic displacement and gas production cross-sections were obtained for a number of materials to calculate radiation damage and gas production rate in nuclear- and fusion reactors, and neutron spallation sources. An advanced atomistic modelling approach was applied for calculations of the number of stable displacements in materials.
\end{abstract}

\section{Introduction}

The evaluation of atomic displacement and gas production cross-sections for irradiated materials is a challenge considering the modelling of nuclear reactions, the simulation of atomic interactions, and the analysis and the use of available experimental data.

The report describes displacement and gas production cross-sections recently evaluated in KIT and the methods used to obtain the evaluated data.

The displacement cross-section for incident particle with the kinetic energy $\mathrm{E}_{p}$ is calculated as follows

$$
\sigma_{d}\left(E_{p}\right)=\sum_{i} \int_{E_{d}}^{T_{i}^{\max }}\left(d \sigma_{i}\left(E_{p}, T_{i}\right) / d T_{i}\right) N_{D}\left(T_{i}\right) d T_{i},
$$

where $d \sigma_{i} / d T_{i}$ is the recoil energy distribution of primary knock-on atoms (PKA) produced in $i$-th nuclear reaction; $N_{D}\left(T_{i}\right)$ is the number of stable defects produced by PKA with the kinetic energy $T_{i}, T_{i}^{\max }$ is the maximal kinetic energy of the PKA in $i$-th reaction; $E_{d}$ is the average threshold displacement energy of material.

The calculation of displacement cross-section assumes the use of nuclear models to get recoil energy distributions and the simulation of atomic collision to obtain the number of stable displacements in irradiated material.

\section{Calculation approach}

Calculations were performed using nuclear models implemented in the MCNP [1], CASCADE [2,3], DISCAC [4], TALYS [5,6], and ALICE/ASH [3,7] codes depending on the task and on the applicability of models at different incident nucleon energy and target ranges. Results obtained using various models and codes were also applied for a verification of calculations and the estimation of the uncertainty of theoretical predictions [8-13].

The calculation of recoil energy distributions is discussed in Ref. [14]. Advanced calculations of gas production components are described in Refs. [6,11,15].

a e-mail: alexander.konobeev@kit.edu
Displacement cross-sections for most materials were obtained in two forms, using the NRT model [16] and the approach, which combines the binary collision approximation model (BCA) and molecular dynamics (MD) simulations $[8,11,17]$.

The BCA calculations were performed using the IOTA [18] and SRIM codes [19] at relative high energies of ions; the available results of MD simulations [17] were utilized at low ion energies to estimate the total number of stable displacements.

The BCA-MD calculations are discussed in details in Refs. [8,11]. An example of calculations is shown in Fig. 1. The figure shows the ratio of the number of stable displacements $N_{D}$ calculated using BCA-MD to the number of defects predicted by the NRT model (defect production efficiency) for $\mathrm{Fe}-\mathrm{Fe}$ irradiation. The calculations were performed with the IOTA code using different screening functions [18] and with the SRIM code $[19,20]$. The results of MD simulations from Ref. [21] were applied. The systematics data were obtained from original data [22].

\section{Atomic displacement cross-sections}

Displacement cross-sections were obtained for different materials and irradiations. A special attention was paid to the uncertainty of calculated cross-sections.

\subsection{Neutron displacement cross-sections for ${ }^{9} \mathrm{Be}$ at energies up to $200 \mathrm{MeV}$}

The evaluation of $\sigma_{d}$ consisted of i) test model calculations of energy and angular particle distributions in proton induced reactions to estimate the "quality" of model predictions and to quantify the deviation between calculated values and measured data, ii) calculations for $\mathrm{n}+{ }^{9} \mathrm{Be}$ reactions, and iii) an adjustment of calculated values to JEFF data below $20 \mathrm{MeV}$. The details are described in Ref. [23]. Figure 2 shows the obtained $\sigma_{d}$ cross-sections.

Numerical data can be found in Ref. [24]. 


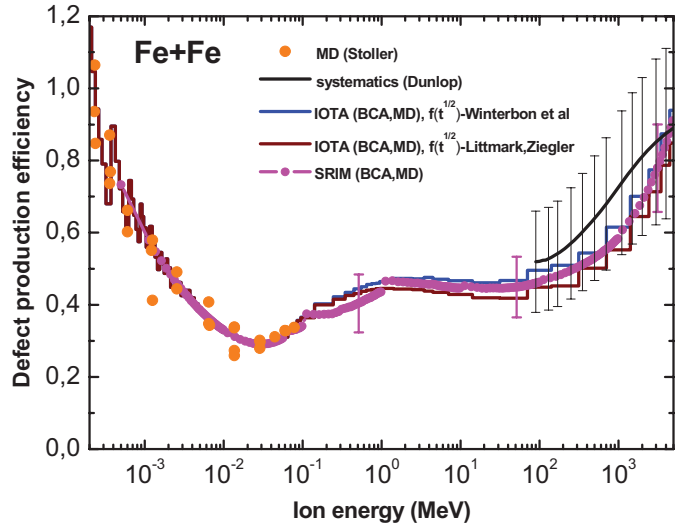

Figure 1. Defect production efficiency for Fe-Fe irradiation calculated with the IOTA code and SRIM code. The $E_{d}$ value is equal to $40 \mathrm{eV}$. See details in the text.

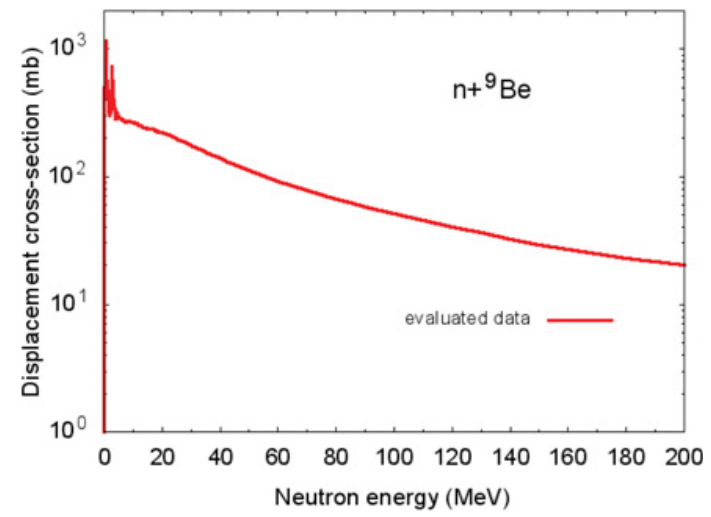

Figure 2. Neutron displacement cross-sections for ${ }^{9} \mathrm{Be}$ obtained using the NRT model. The $E_{d}$ energy is equal to $31 \mathrm{eV}$.

\subsection{Displacement cross-sections for EUROFER}

The calculations of displacement cross-sections values were performed using the recoil energy distributions obtained from neutron data libraries as discussed in Ref. [25] and results of BCA-MD simulations with the IOTA code. The number of stable displacements was calculated for main components of EUROFER considering PKAs moving in stainless steel in contrast to the usual procedure, where $\sigma_{d}$ is calculated as a weighted sum of the independent components [25].

The evaluation procedure consisted of the removing of possible peculiarities in $\sigma_{d}$ values resulting from the use of $d \sigma / d T$ taken from neutron data libraries, especially at $20 \mathrm{MeV}$, the fitting to results of $\sigma_{d}$ calculations using intranuclear cascade evaporation models above $150 \mathrm{MeV}$, and the combination of the different results below and above $20 \mathrm{MeV}$, if necessary. Obtained values are shown in Fig. 3 both for BCA-MD and NRT model.

Data are available in Ref. [24].

\subsection{Evaluated data at incident nucleon energies up to $3 \mathrm{GeV}$ and higher}

Nuclear data used for the calculation of recoil energy distributions at low incident neutron energies $[8,11]$ were taken from ENDF/B-VII and processed using the NJOY code [26]. At higher incident energies recoil spectra were calculated using appropriate models: the model describing the scattering of charged particles in the matter, the optical

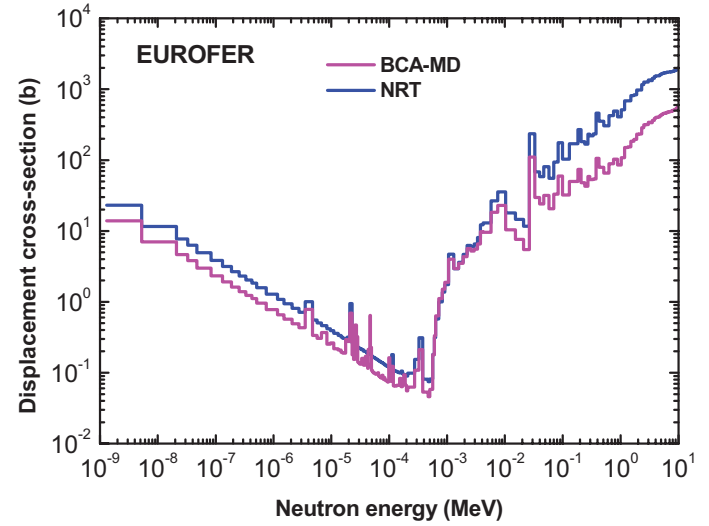

Figure 3. Displacement cross-sections for EUROFER obtained using the BCA-MD approach and the NRT model. The $E_{d}$ value is equal to $40 \mathrm{eV}$.

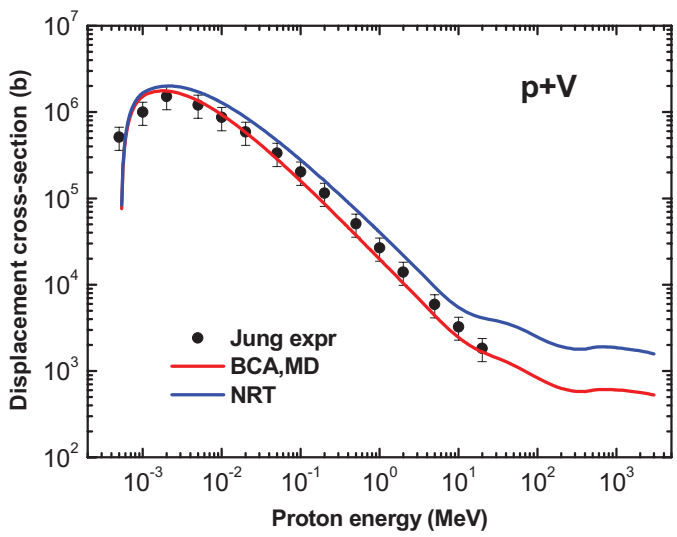

Figure 4. Displacement cross-section for $\mathrm{p}+\mathrm{V}$ interactions.

model, the pre-equilibrium model, and the intranuclear cascade evaporation model. At intermediate energies of primary particles the reliability of obtained displacement cross-sections was improved by using of weighted results of calculations obtained by different approaches, see details in Refs. $[8,10,11]$. The numbers of stable defects in irradiated materials were calculated using the BCA-MD approach.

Figure 4 shows the example of obtained displacement cross-sections for proton irradiation of vanadium. Experimental data are taken from Ref. [27].

The evaluated displacement cross-sections were obtained for $\mathrm{Al}, \mathrm{Ti}, \mathrm{V}, \mathrm{Cr}, \mathrm{Fe}, \mathrm{Ni}, \mathrm{Cu}, \mathrm{Zr}$, and $\mathrm{W}$ irradiated with neutrons and protons at energies from $10^{-5} \mathrm{eV}$ to $3 \mathrm{GeV}[10,28]$. Data in ENDF-6 format can be found in Ref. [28].

Data for $\mathrm{Fe}, \mathrm{Cu}$, and $\mathrm{W}$ were obtained at primary proton energies up to $100 \mathrm{GeV}$ [8].

\subsection{Study of uncertainty of cross-sections}

The uncertainties of calculated displacement crosssections were analysed at incident neutron energies above $0.1 \mathrm{MeV}$ using the Monte Carlo method [29].

Both the NRT model and the arc-dpa approach [30] were applied for estimation of the number of stable displacements. The four parameters, including $\mathrm{E}_{d}$, were varied while using NRT model and two parameters when using the arc-dpa approach $[12,13]$. 


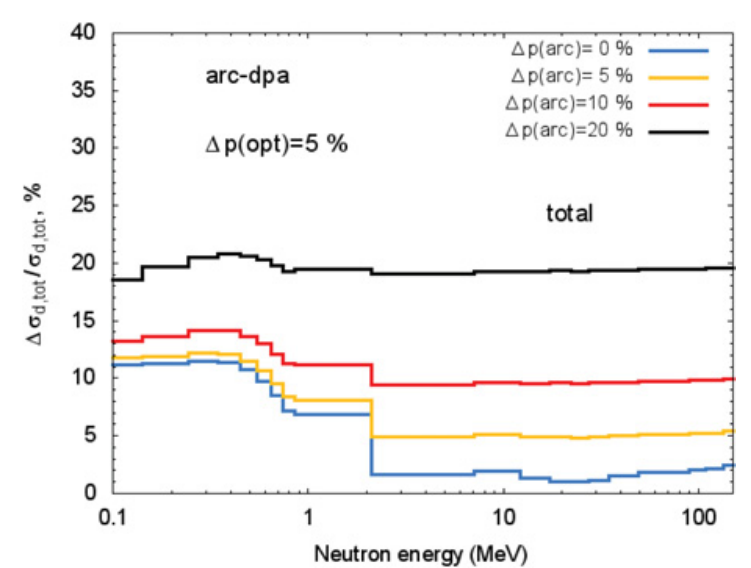

Figure 5. The RSD values of displacement cross-sections for iron calculated using the arc-dpa approach [30] with different variation of parameters and the variation of optical model and nuclear level density parameters with the RSD values equal to $5 \%$ and $10 \%$, correspondingly. See details in Refs. $[12,13]$.

Figure 5 shows the example of estimated relative standard deviation (RSD) of displacement cross-sections.

\section{Gas production cross-sections}

\subsection{Evaluation of atomic mass dependence of components of gas production cross-sections}

By analogy with the evaluation of the energy dependence of cross-sections, atomic mass dependence (A) of gas production cross sections was evaluated for a number of incident nucleon energies. The choice of the incident energy depends on available experimental data. The evaluation procedure of A-dependence is discussed in Refs. [31,32].

The proton-, deuteron-, triton-, ${ }^{3} \mathrm{He}-$, and $\alpha$-particle production cross-sections were obtained for 278 stable targets from ${ }^{7} \mathrm{Li}$ to ${ }^{209} \mathrm{Bi}$ at proton incident energies 62,90 , $150,600,800$, and $1200 \mathrm{MeV}[31,32]$ and at the neutron incident energy equal to $96 \mathrm{MeV}$ [15].

Figure 6 shows the example of evaluated $\alpha$-particle production cross-section as a function of the target atomic mass number.

The obtained cross-sections [15,30,31] can be used as the "reference points" for data evaluation for targets where experimental data are rare or missing.

\subsection{Gas production data for Be and target nuclei from $\mathrm{Mg}$ to $\mathrm{Bi}$ at neutron incident energies up to $200 \mathrm{MeV}$}

Proton-, deuteron-, triton-, ${ }^{3} \mathrm{He}$-, and $\alpha$-particles- production cross-sections were obtained for beryllium and other 262 stable nuclides with atomic number from 12 to 83 at the energies of primary neutrons up to $200 \mathrm{MeV}$.

The data evaluation consisted of the analysis of available experimental data, the estimation of atomic mass dependence of cross-sections to improve final evaluated curves, the analysis of evaluated data from ENDF/B-VII.1, JENDL-4, JEFF-3.2, and TENDL-2014, nuclear model calculations, the improvement of existing evaluated data concerning the incorrect energy dependence, and statistical combination of experimental and theoretical data. The

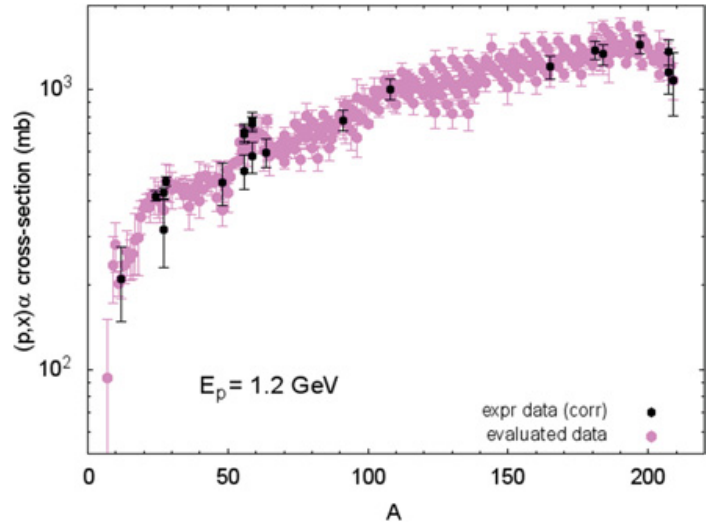

Figure 6. Evaluated $\alpha$-particle production cross-sections for 278 stable target nuclei at the incident proton energy $1.2 \mathrm{GeV}$. Experimental data are overviewed in Ref. [31].

detail description of the evaluation and the data is given in Ref. [15,23].

Data in ENDF-6 format are available in Ref. [24].

\subsection{Evaluated data at incident nucleon energies up to $3 \mathrm{GeV}$}

The evaluation was performed using results of nuclear model calculations, available experimental data, and systematic predictions.

Evaluated proton-, deuteron-, triton-, ${ }^{3} \mathrm{He}-$, and $\alpha$-particles- production cross-sections were obtained for $\mathrm{Be}, \mathrm{Al}, \mathrm{Ti}, \mathrm{Cr}, \mathrm{Fe}, \mathrm{Ni}$, and $\mathrm{W}$ irradiated with nucleons with energies from $10^{-5} \mathrm{eV}$ to $3 \mathrm{GeV}[10,28,33]$.

Data in ENDF-6 format for $\mathrm{Ti}, \mathrm{Cr}, \mathrm{Fe}, \mathrm{Ni}$, and $\mathrm{W}$ can be found in Ref. [28].

\section{Conclusion}

Atomic displacement cross-sections were obtained for Be, $\mathrm{Al}, \mathrm{Ti}, \mathrm{V}, \mathrm{Cr}, \mathrm{Fe}, \mathrm{Ni}, \mathrm{Cu}, \mathrm{Zr}$, and $\mathrm{W}$ to estimate the radiation damage and gas production rates in nuclear- and fusion reactors, and neutron spallation sources. The NRT model and an advanced atomistic modelling approach combining the use of binary collision approximation model and results of molecular dynamics simulations were utilized for calculations of the number of stable displacements in materials.

Proton-, deuteron-, triton-, ${ }^{3} \mathrm{He}$, and $\alpha$-particle production cross-sections were evaluated for 278 stable target nuclei from $\mathrm{Li}$ to $\mathrm{Bi}$ irradiated with intermediate and high energy nucleons using available experimental information and results of model calculations.

Obtained numerical data are partly available in Refs. [24, 28].

The work leading to this publication has been funded partially by Fusion for Energy under the Specific Grant Agreements F4EGRT-168.01 and F4E-GRT-168.02. This publication reflects the views only of the authors, and Fusion for Energy cannot be held responsible for any use which may be made of the information contained therein. 


\section{References}

[1] J.T. Goorley, M.R. James, T.E. Booth, F.B. Brown, et al. Ed. D.B. Pelowitz, LA-CP-13-00634, 2013

[2] V.S. Barashenkov, Comp. Phys. Com. 126, 28 (2000)

[3] A.Yu. Konobeyev, U. Fischer, Simulation of heavy cluster emission in nucleon induced reactions on targets from $C$ to Bi at intermediate energies, Report KIT SR 7684, 2014; http://goo.gl/tYkolF; http://digbib.ubka.uni-karlsruhe.de/ volltexte/1000043611

[4] C.H.M. Broeders, A.Yu. Konobeyev, Yu.A. Korovin, V.N. Sosnin, DISCA code system, FZKA 7221, 2006; http://d-nb.info/98154746x/34

[5] A.J. Koning, S. Hilaire, M.C. Duijvestijn, TALYS, Proc. ND2007, Nice, 2007, p. 211; TALYS-1.8, http://www.talys.eu/

[6] A.Yu. Konobeyev, U. Fischer, P.E. Pereslavtsev, A. Koning, M. Blann, Implementation of GDH model in TALYS-1.7 code, Report KIT SWP 45, 2016, http://digbib.ubka.uni-karlsruhe.de/ volltexte/1000052543

[7] C.H.M. Broeders, A.Yu. Konobeyev, A.Yu. Korovin, V.P. Lunev, M. Blann, ALICE/ASH - Pre-compound and evaporation model code system, FZKA 7183, 2006, http://d-nb.info/980834465/34

[8] A.Yu. Konobeyev, U. Fischer, C.H.M. Broeders, L. Zanini, Proc. AccApp'07, Pocatello, Idaho, 2007; http://mathematicsandcomputation. cowhosting.net/ACCAPP-2007/data/papers/ 178099.pdf

[9] A.Yu. Konobeyev, U. Fischer, Proc. NEMEA-4 Prague, 2007, p. 51 (available online)

[10] A.Yu. Konobeyev, U. Fischer, C.H.M. Broeders, L. Zanini, IAEA-NDS-214, 2009; https://wwwnds. iaea.org/publications/iaea-nds/iaeands-0214.pdf

[11] A.Yu. Konobeyev, U. Fischer, L. Zanini, Proc. AccApp'11, Knoxville,2011; https://goo.gl/ aX2DuQ

[12] A.Yu. Konobeyev, U. Fischer, S.P. Simakov, In: INDC(NDS)-0719, 2016, to be published

[13] A.Yu. Konobeyev, U. Fischer, S.P. Simakov, Uncertainties of displacement cross-sections for iron and tungsten at neutron irradiation energies above 0.1 MeV, KIT SWP 49, 2016; http://goo.gl/ p9ur50; http://digbib.ubka.uni-karlsruhe. de/volltexte/1000057548

[14] A.Yu. Konobeyev, U. Fischer, INDC(NDS)0691, 2015, p.27; https://goo.gl/UOZjC7; https://www-nds.iaea.org/publications/ indc/indc-nds-0691.pdf

[15] A.Yu. Konobeyev, U. Fischer, Complete gas production data library for nuclides from $\mathrm{Mg}$ to $\mathrm{Bi}$ at neutron incident energies up to $200 \mathrm{MeV}$, Report KIT SWP 36, 2015; http://goo.gl/F8hKLN; http://digbib.ubka.uni-karlsruhe.de/ volltexte/1000049466

[16] M.J. Norgett, M.T. Robinson, I.M. Torrens, Nucl. Eng. Des. 33, 50 (1975)

[17] A.Yu. Konobeyev, U. Fischer, Proc. HB2014, EastLansing, MI, 2014, p.433; https://goo.gl/ BqXBG6; http://accelconf .web.cern.ch/ AccelConf/HB2014/papers/tho4ab02.pdf

[18] C.H.M. Broeders, A.Yu. Konobeyev, K. Voukelatou, IOTA - a code to study ion transport and radiation damage, FZKA 6984 2004; http://d-nb.info/ 972618937/34; http://goo.gl/4MALd9

[19] J.F. Ziegler, SRIM; http://srim.org/

[20] A.Yu. Konobeyev, U. Fischer, S.P. Simakov, https://www-nds . iaea.org/CRPdpa/SRIM_MD . pdf

[21] R. E. Stoller, Nucl. Eng. Des. 195, 129 (2000)

[22] A. Dunlop, D. Lesueur, P. Legrand, H. Dammak, Nucl. Instr. Meth. B90, 330 (1994)

[23] A.Yu. Konobeyev, U. Fischer, Evaluation of atomic displacement and gas production cross-section for ${ }^{9}$ Be irradiated with neutrons at energies up to 200 MeV, KIT SWP 37, 2015; http: //goo.gl/3zoyND; http://digbib.ubka.uni-karlsruhe.de/ volltexte/1000049467

[24] Evaluated atomic displacement and gas production cross-sections at intermediate nucleon energies, https://bwsyncandshare.kit.edu/dl/ fiFWHQ2eVYVFRCqd3S9CxjFU/data_INR_KIT . zip https ://1drv.ms/u/s! Ahx7kBUbWe3A6CgxLe0 qApND2bGs

[25] A.Yu. Konobeyev, U. Fischer, P.E. Pereslavtsev, Kerntechnik 80, 7 (2015)

[26] http://t2.lanl.gov/nis/codes/NJOY12/ index.html

[27] P. Jung, J. Nucl. Mater. 117, 70 (1983)

[28] https://www-nds.iaea.org/public/downloadendf/DXS

[29] D.L. Smith, Covariance matrices for nuclear cross sections derived from nuclear model calculations, ANL/NDM-159, 2004

[30] K. Nordlund, INDC(NDS)-0691, 2015, p. 19; See Ref. [14]

[31] A.Yu. Konobeyev, U. Fischer, Reference data for evaluation of gas production cross-sections in proton induced reactions at intermediate energies, KIT SR 7660, 2014, http://goo.gl/26Vjzl; http: // www.ksp.kit.edu/download/1000038463

[32] A.Yu. Konobeyev, U. Fischer, Kerntechnik 80, 90 (2015)

[33] S. Akça, A.Yu. Konobeyev, U. Fischer, Kerntechnik 79, 464 (2014) 